\title{
Erratum to: Pathologic Complete Response to Intralesional Interleukin-2 Therapy Associated with Improved Survival in Melanoma Patients with In-Transit Disease
}

Saima Hassan, MD, PhD, FRCSC ${ }^{1,2}$, Teresa M. Petrella, MD, MSc, FRCPC ${ }^{3}$, Tong Zhang, MSc $^{4}$, Suzanne Kamel-Reid, BA, MA, PhD, FACMG ${ }^{4}$, Francesco Nordio, PhD $^{5}$, Andrea Baccarelli, MD, PhD ${ }^{6}$, Shachar Sade, MD, MSc, FRCPC ${ }^{7}$, Karen Naert, MD, FRCPC ${ }^{4}$, Ayman Al Habeeb, MBBS, FRCPC ${ }^{4}$, Danny Ghazarian, MB ChB, PhD, FRCPC, FCAP ${ }^{4}$, and Frances C. Wright, MD, MEd, FRCSC ${ }^{1}$

${ }^{1}$ Division of Surgical Oncology, Sunnybrook Health Sciences Centre, Odette Cancer Centre, Toronto, ON, Canada; ${ }^{2}$ Department of Biomedical Engineering, Oregon Health and Science University, Portland, OR; ${ }^{3}$ Division of Medical Oncology, Sunnybrook Health Sciences Centre, Toronto, ON, Canada; ${ }^{4}$ Department of Pathology, University Health Network, Toronto, ON, Canada; ${ }^{5}$ Department of Medicine, Brigham and Women's Hospital, Boston, MA; ${ }^{6}$ Department of Environmental Health and Epidemiology, Harvard School of Public Health, Boston, MA; ${ }^{7}$ Department of Pathology, Sunnybrook Health Sciences Centre, Toronto, ON, Canada

\section{ERRATUM TO: ANN SURG ONCOL} DOI 10.1245/S10434-014-4199-Z

In the original article, the first sentence in the Results section of the abstract is incorrect. The corrected sentence is as follows:
Ten patients $(10 / 31,32.3 \%)$ achieved a pathologic complete response (pCR), 17/31 (54.8\%) had a partial response, and 4/31 (12.9\%) had progressive disease on treatment.

The online version of the original article can be found under doi:10. 1245/s10434-014-4199-z.

(C) Society of Surgical Oncology 2014

Published Online: 12 December 2014

F. C. Wright, MD, MEd, FRCSC

e-mail: frances.wright@sunnybrook.ca 